\title{
Notas sobre la variación de briófitas entre un bosque pantanoso de Amomyrtus luma y una pradera antropogénica húmeda de Juncus procerus
}

\author{
Notes on the variation of bryophytes between a swamp forest dominated by Amomyrtus \\ luma and an anthropogenic wet prairie of Juncus procerus
}

\author{
Víctor Ardiles ${ }^{1}$, Alvaro Promis ${ }^{2, *}$, Daniela Cortés ${ }^{2}$ \\ 1Área de Botánica, Museo Nacional de Historia Natural, Quinta Normal, Santiago, Chile. \\ ¿2Universidad de Chile, Facultad de Ciencias Forestales y de la Conservación de la Naturaleza, Departamento de Silvicultura y Conservación de la Naturaleza, Santa Rosa 11315, La \\ Pintana, Santiago, Chile \\ *E-mail: alvaro.promis@gmail.com
}

\section{ABSTRACT}

The bryophyte richness was evaluated in the understory of a swamp forest of Amomyrtus luma and an anthropogenic wet prairie of Juncus procerus. Bryophyte richness, and growth and life forms changed between both wetlands. Because of differences between bryophyte species, both kind of wetlands should be considered for conservation efforts.

Las briófitas utilizan hábitats con particulares condiciones microclimáticas, respecto a humedad, temperatura y luminosidad (León-Vargas et al. 2006, Stewart \& Mallik 2006). También utilizan diferentes sustratos para establecerse, tales como madera en descomposición, superficies rocosas sombrías, suelo mineral y corteza de árboles; debido a diferencias en el pH, la exposición de la superficie (respecto al sol o viento) y la humedad (Fenton et al. 2015). Los disturbios que modifican el microhábitat de establecimiento pueden ejercer cambios en la presencia o ausencia de briófitas, tanto en bosques (Frego 2007, Fenton et al. 2015) como en humedales (Stapanian et al. 2016), lo que puede estar influido por sus rasgos funcionales (Baldwin \& Bradfield 2007, Fenton et al. 2015).

Los bosques pantanosos son humedales, en los que su fisonomía está determinada por la presencia de especies arbóreas y arbustivas, que crecen sobre suelos generalmente inundados por agua y pobremente aireados (Ramírez et al. 1996, Correa-Araneda et al. 2011). En Chile, se ha descrito la existencia de bosques pantanosos desde la región de Coquimbo hasta la de Magallanes (Pisano 1977, Ramírez et al. 1996, Correa-Araneda et al. 2011). Estos bosques pantanosos han sido mayoritariamente estudiados desde un punto de vista de la vegetación y flora vascular que sustentan (Correa-Araneda et al. 2011), y son escasos los trabajos con relevamientos con especies briófitas en sus catálogos florísticos (i.e. Ramírez et al. 1996).

A lo largo de Chile estos bosques pantanosos han sido objeto de alteraciones antrópicas, especialmente para el desarrollo de actividades agropecuarias y forestales (Correa-Araneda et al. 2011, Promis et al. 2013). Estas alteraciones transforman la estructura y composición de estos humedales hacia una fase palustre de la hidrosere, en la que las especies arbóreas pierden su dominancia (Ramírez et al. 1996, Promis et al. 2013). Estos disturbios modifican condiciones de microhábitat (i.e. microclima y sustrato), lo que produce cambios en los grupos de especies de briófitas que se establecen (Spatanien et al. 2016).

El objetivo principal de este trabajo fue el de evaluar el cambio en la riqueza de especies de briófitas, a nivel de especies y de algunas estrategias de vida, en dos tipos de humedales: un bosque pantanoso y una pradera

Open Access Journal

(02020 The author(s). Gayana Botánica (2020 Universidad de Concepción. This open access article is distributed under the terms of the Creative Commons Attribution-NonCommercial 4.0 International License which permits any noncommercial use, distribution, and reproduction, as long as you give appropriate credit to the original author(s) and the source. 
antropogénica húmeda de Juncus procerus E. Mey. Ambas superficies se encuentran contiguas y ubicadas a unos $20 \mathrm{~km}$ al este de Puerto Cisnes ( $44^{\circ} 42^{\prime}$ S; $72^{\circ} 33^{\prime}$ O; 60 m.s.n.m.). El bosque pantanoso (15 ha) tiene una densidad de 1.168 árboles/ha y un área basal de $26,8 \mathrm{~m}^{2} / \mathrm{ha}$. $60,3 \%$ de los árboles corresponden a Amomyrtus luma (Molina) D. Legrand et Kausel y $53,9 \%$ del área basal a Nothofagus nitida (Phil.) Krasser (Promis et al. 2013). La pradera húmeda (13 ha) era parte del bosque pantanoso, pero durante la época de 1960 el bosque fue cortado y quemado para un uso ganadero (Promis et al. 2013).

En abril de 2010 se muestreó la presencia de especies de briófitas en seis parcelas de $6 \mathrm{~m}^{2}(2 \times 3 \mathrm{~m})$ en cada humedal, las que fueron aleatoriamente distribuidas (Promis et al. 2013). En cada una de estas parcelas se muestreó la presencia de todas las especies briófitas hasta una altura de dos metros. Se recolectaron muestras de todas las especies briófitas para determinar su identidad en laboratorio, con el apoyo de literatura especializada, basado en el análisis macro y micromorfológico de los gametofitos y esporofitos, siempre que estuvieran presentes. Las especies briófitas fueron clasificadas en grupos funcionales, considerando la siguiente separación: taxonomía (Bryophyta, Marchantiophyta y Anthocerotophyta); morfología (acrocarpo y pleurocarpo para Bryophyta; folioso y taloso simple para Marchantiophyta y taloso para Anthocerotophyta) y formas de vida (abanico, césped corto, césped largo, colgante, dendroide, estera filiforme, estera suave, estera taloide y trama) (siguiendo a Glime 2017). Además, para cada muestra de especie briófita se clasificó el sustrato sobre el que se encontraba establecida (corteza de árbol, hoja, rama o suelo).

La nomenclatura para las especies de Bryophyta sigue la propuesta por Müller (2009), cuyas familias se revisaron de acuerdo con Goffinet \& Shaw (2009) y Tropicos.org (2019). Para las Marchantiophytas y Anthocerotophytas se siguió a Söderström et al. (2016) y Hässel de Menéndez \& Rubies (2009). La verificación de las especies se comparó con ejemplares de herbario (SGO, Chile y BA, Argentina) y el material estudiado se depositó en el Herbario del Museo Nacional de Historia Natural (SGO). Se determinó el patrón de distribución global y nacional (local) de las especies, considerando la bibliografía utilizada para la determinación de las especies, además de las propuestas establecidas por Villagrán \& Barrera (2002), Villagrán et al. (2002), Villagrán et al. (2005) y León et al. (2014).

Se registraron 61 especies briófitas en ambos ambientes, de las que 19 especies $(31,1 \%)$ corresponden a la división Bryophyta, 41 especies $(67,2 \%)$ a la división Marchantiophyta y una especie $(1,6 \%)$ a la división Anthocerotophyta. El total de plantas se distribuyó en 32 familias y 42 géneros. Las familias con mayor cantidad de especies fueron Dicranaceae para la división Bryophyta y Lophocoleaceae para Marchantiophyta. La familia Dendrocerotaceae es la única presente en la división Anthocerotophyta. La mayoría de los géneros $(76,2 \%)$ están representados solamente por una especie (Anexo 1).

Desde un punto de vista de distribución global, 44 especies $(72,1 \%)$ son endémicas del cono sur de Sudamérica. Específicamente en Chile, una gran mayoría de las especies presenta una distribución Valdiviana - Norpatagónica y Subantártica (VNS) (Anexo 1). Ambos patrones se evidencian también al interior de las divisiones taxonómicas.

En el bosque pantanoso se encontraron 41 especies briófitas y en la pradera húmeda 39 especies, de las que 19 son comunes. La riqueza de especies briófitas en el bosque pantanoso $(20,3 \pm 1,8$ especies/parcela; promedio \pm error estándar) fue estadísticamente mayor a la registrada en la pradera húmeda (14,0 \pm 3,6 especies/parcela) (prueba $U$ de Mann-Whitney; $U=1,5 ; p<0,008$ ), patrón que también se presentó al analizar la riqueza de especies a nivel de divisiones, siendo mayor la riqueza de especies de Anthocerotophyta y Marchantiophyta en el bosque pantanoso respecto a la pradera húmeda (Tabla 1).

En cuanto a la forma de crecimiento de las briófitas, en la división Bryophyta se registraron las morfologías acrocarpo y pleurocarpo (Tabla 1). Las plantas acrocárpicas aumentaron en riqueza de especies en la pradera húmeda respecto al bosque pantanoso, y la riqueza de especies de plantas pleurocárpicas fue mayor en el bosque pantanoso respecto a la pradera húmeda. En la división Marchantiophyta se registraron dos tipos de morfologías, la talosa simple y la foliosa, siendo las primeras las que presentaron una menor riqueza de especies en la pradera húmeda (Tabla 1). La única planta con hábito taloso (división Anthocerotophyta) fue encontrada en el bosque pantanoso (Tabla 1).

En el bosque pantanoso se encontró una mayor riqueza de especies en la mayoría de las categorías de formas de vida respecto a la pradera húmeda, especialmente para las formas de vida abanico, estera filiforme y estera taloide (prueba de Mann-Whitney, $p<0,05$ ). Mientras que en la pradera húmeda las formas de vida tipo césped presentaron mayor riqueza de especies respecto al bosque pantanoso, aunque esta diferencia no fue estadísticamente significativa (prueba de Mann-Whitney, $p>0,05$ ) (Tabla 1).

En término de sustrato, más del $50 \%$ de las especies presentes en el bosque pantanoso y en la pradera húmeda 
mostraron preferencia por establecerse sobre corteza de árboles y en segunda preferencia sobre el suelo (entre el 28 y $32 \%$ ) (Fig. 1).

Los resultados expuestos resaltan la alta riqueza de especies briófitas en humedales de la zona austral del país y con rasgos biogeográficos de alto endemismo del cono sur de Sudamérica, patrón que converge con los antecedentes de aumento de especies y endemismo de briófitas hacia el sur del país (Shaw et al. 2005, Rozzi et al. 2008, León et al. 2014, Villagrán 2018).

Las diferencias de especies briófitas encontradas entre ambos ambientes mantienen el patrón de cambio de la vegetación de plantas vasculares anteriormente expuesto por Promis et al. (2013), debido a la quema y corta del bosque pantanoso, para un uso de actividad agropecuaria, lo que produjo la formación de la pradera húmeda. Específicamente en este estudio se muestra un patrón de disminución de la riqueza de especies y grupos funcionales de plantas briófitas debido al cambio de estructura y composición del bosque pantanoso a una pradera húmeda. Resultados similares fueron encontrados también al evaluar la composición florística de especies entre turberas naturales y antropogénicas en Chiloé, siendo aquellas que presentan extracción de musgo las que muestran un mayor cambio (Díaz et al. 2008).

TABLA 1. Riqueza de especies de briófitas ( $n^{\circ}$ de especies promedio por parcela \pm error estándar) en el bosque pantanoso (BP) y en la pradera antropogénica húmeda de Juncus procerus ( $\mathrm{PHJ}$ ), para todas las plantas por parcela, por división taxonómica, morfología y forma de vida. Entre paréntesis, el valor estadístico de la prueba $\mathrm{U}$ de Mann-Whitney. ${ }^{*}=$ diferencia significativa a $p<0,05$. / Bryophyte species richness (averaged number of species per plot \pm standard error) in the swamp forest (BP) and in the anthropogenic wet prairie of Juncus procerus (PHJ), for the overall species per plot, taxonomic classification, morphology and life form. In brackets the statistic value of the Mann-Whitney $\mathrm{U}$ test. ${ }^{*}=$ significant differences at $p<0.05$.

\begin{tabular}{|c|c|c|c|}
\hline Riqueza Especies & $\mathrm{BP}$ & PHJ & Prueba U Mann-Whitney \\
\hline Todas las Briófitas (61) & $20,3 \pm 1,8$ & $14,0 \pm 3,6$ & $1,5(0,008)^{*}$ \\
\hline \multicolumn{4}{|l|}{ División Taxonómica } \\
\hline Anthocerotophyta (1) & $0,8 \pm 0,4$ & $0,0 \pm 0,0$ & $3,0(0,005)^{*}$ \\
\hline Bryophyta (19) & $6,0 \pm 2,0$ & $5,3 \pm 1,2$ & $14,0(0,589)$ \\
\hline Marchantiophyta (41) & $13,5 \pm 3,0$ & $8,7 \pm 2,9$ & $4,0(0,024)^{*}$ \\
\hline \multicolumn{4}{|l|}{ Morfología } \\
\hline Taloso (1) & $0,8 \pm 0,4$ & $0,0 \pm 0,0$ & $3,0(0,005)^{*}$ \\
\hline Acrocarpo (9) & $1,3 \pm 0,5$ & $3,5 \pm 0,8$ & $0,0(0,003)^{*}$ \\
\hline Pleurocarpo (10) & $4,7 \pm 1,8$ & $1,8 \pm 1,5$ & $3,5(0,019)^{*}$ \\
\hline Foliosa (37) & $12,2 \pm 2,6$ & $8,5 \pm 2,6$ & $6,0(0,051)$ \\
\hline Talosa simple (4) & $1,3 \pm 0,5$ & $0,2 \pm 0,4$ & $2,0(0,006)^{*}$ \\
\hline \multicolumn{4}{|l|}{ Forma de Vida } \\
\hline Abanico (11) & $5,5 \pm 0,8$ & $3,2 \pm 0,8$ & $0,0(0,003)^{*}$ \\
\hline Colgante (1) & $0,5 \pm 0,5$ & $0,0 \pm 0,0$ & $9,0(0,056)$ \\
\hline Césped corto (9) & $2,2 \pm 1,0$ & $3,3 \pm 1,5$ & $10,0(0,175)$ \\
\hline Césped largo (7) & $1,7 \pm 0,5$ & $1,8 \pm 1,5$ & $18,0(1,000)$ \\
\hline Dendroide (6) & $2,2 \pm 0,8$ & $1,2 \pm 0,8$ & $6,5(0,051)$ \\
\hline Estera filiforme (3) & $1,5 \pm 0,5$ & $0,3 \pm 0,5$ & $3,0(0,011)^{*}$ \\
\hline Estera suave (13) & $3,0 \pm 1,3$ & $2,5 \pm 1,8$ & $14,5(0,548)$ \\
\hline Estera taloide (3) & $1,2 \pm 0,8$ & $0,0 \pm 0,0$ & $3,0(0,007)^{*}$ \\
\hline Trama (8) & $2,7 \pm 0,5$ & $1,7 \pm 1,6$ & $11,0(0,241)$ \\
\hline
\end{tabular}




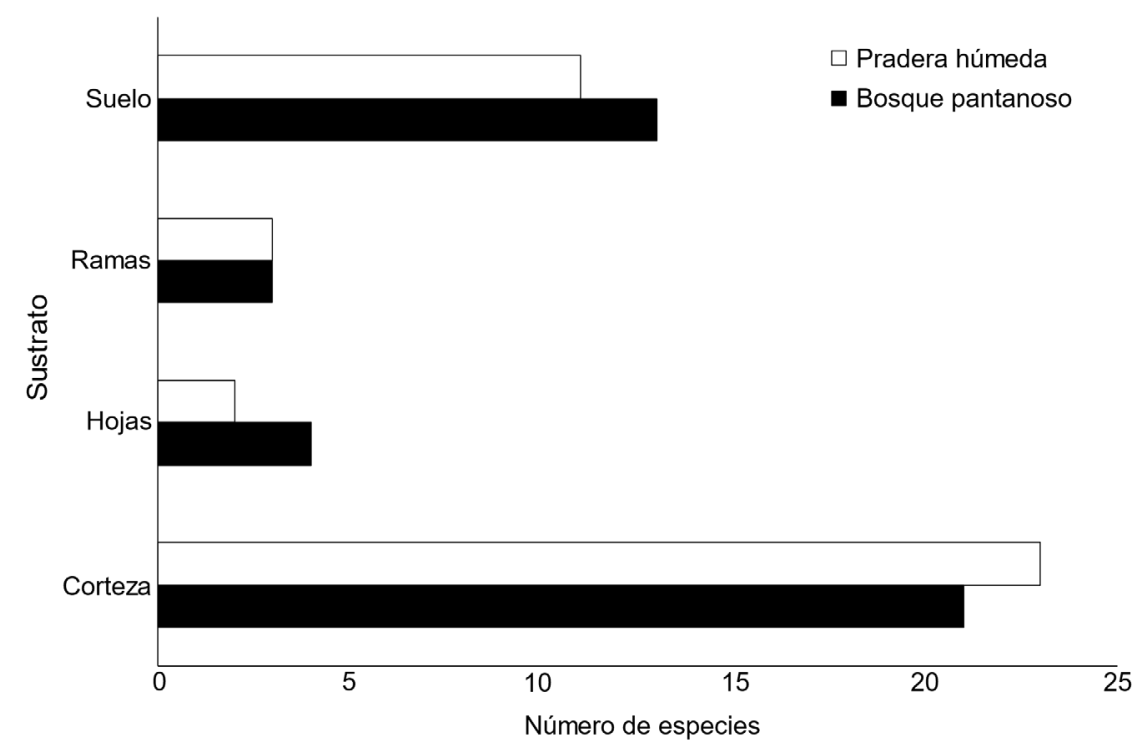

Figura 1. Número de especies de briófita s distribuidas por sustrato de preferencia en el bosque pantanoso y en la pradera antropogénica húmeda de Juncus procerus. / Number of species per substrate preferences in the swamp forest and in the anthropogenic wet prairie of Juncus procerus.

Desde un punto de vista microclimático, la radiación solar y el viento dentro de un bosque disminuyen respecto a una situación abierta, la oscilación térmica entre el día y la noche es menor y la humedad del aire es mayor (Donoso et al. 2014). Esto influye en la mayor riqueza de especies briófitas que prevalecen en el ambientes más húmedo y sombrío del bosque pantanoso respecto a la pradera húmeda, como son las especies de hábito más rastrero, tales como aquellas pertenecientes a las divisiones Marchantiophyta (Frahm 2002) y Anthocerotophyta, las especies briófitas con forma de crecimiento pleurocárpico y las formas de vida de abanico, estera filiforme y estera talosa (Bates 1998, Stewart \& Malik 2006, Glime 2017). Mientras que, en la pradera húmeda, donde la radiación solar debiese ser mayor y la humedad del aire menor, se vieron favorecidas especies briófitas con morfología acrocárpica y formas de vida de césped (Bates 1998, Stewart \& Malik 2006, Glime 2017), similar a lo observado en condiciones abiertas y expuestas de bosques de Nothofagus antarctica (G. Forst.) Oerst. (Schaumann 2005).

La conservación de bosques pantanosos debiese ser una tarea importante, especialmente cuando ellos sustentan una alta riqueza de plantas vasculares y no vasculares (Ramírez et al. 1996, Promis et al. 2013) y todavía presentan una alta susceptibilidad a ser transformados hacia otro tipo de uso (Correa-Araneda et al. 2011). La conservación de estos ambientes también debiese considerar una mirada de paisaje, pues sitios alterados, como la pradera húmeda, sustentan especies briófitas y formas de vida y crecimiento que no se encuentran en el interior del bosque pantanoso. En el bosque pantanoso las especies podrían ser más sensibles a las condiciones microclimáticas y en la pradera húmeda más resilientes a los disturbios (Fenton et al. 2015). Se deben plantear esfuerzos en aumentar estudios de largo plazo en monitoreo de especies briófitas y su ecología, pues presentan un alto potencial para ser utilizadas como indicadores de integridad de ecosistemas (Frego 2007, Fenton et al. 2015).

\section{AGRADECIMIENTOS}

Se agradece al proyecto FPA "Conservación de mallines. Una alternativa para proteger humedales, educación ambiental y desarrollo de actividades ecoturísticas".

\section{REFERENCIAS}

Baldwin, L.K., Bradfield, G.E. 2007. Bryophyte responses to fragmentation in temperate coastal rainforests: A functional group approach. Biological Conservation 136: 408-422.

Bates, J.W. 1998. Is 'life-form' a useful concept in bryophyte ecology? Oikos 82: 223-237.

Correa-Araneda, F., Urrutia, J., Figueroa, R. 2011. Estado del conocimiento y principales amenazas de los humedales 
boscosos de agua dulce de Chile. Revista Chilena de Historia Natural 84: 325-340.

Díaz, M.F., Larraín, J., Zegers, G., Tapia, C. 2008. Caracterización florística e hidrológica de turberas de la Isla Grande de Chiloé, Chile. Revista Chilena de Historia Natural 81: 455-468.

Donoso, C., Promis, A., Coopman, R. 2014. El clima. In: Donoso, C., González, M.E., Lara, A. (Eds.), Ecología Forestal. Bases para el manejo sustentable y conservación de los bosques nativos de Chile, pp. 25-131, Ediciones UACh, Valdivia, Chile.

Fenton, N.J., Hylander, K., Pharo, E.J. 2015. Bryophytes in forest ecosystems. In: Peh, K.S.-H., Corlett, R.T., Bergeron, Y. (Eds.), Routledge Handbook of Forest Ecology, pp. 239249. Routledge, Abingdon, UK.

Frahm, J.-P. 2002. Ecology of bryophytes along altitudinal and latitudinal gradients in Chile. Studies in austral temperate rain forest bryophytes 16. Tropical Bryology 21: 67-79.

Frego, K.A. 2007. Bryophytes as potential indicators of forest integrity. Forest Ecology and Management 242(1): 65-75.

Glime, J.M. 2017. Adaptive Strategies: Growth and Life Forms. In: Glime, J.M. (Ed.), Bryophyte Ecology. Volume 1, Chapter 4-5, Ebook sponsored by Michigan Technological University and the International Association of Bryologists. URL: http://digitalcommons. mtu.edu/bryophyte-ecology/

Goffinet, B., Shaw, J. 2009. Bryophyte Biology. Cambridge University Press, Cambridge. 565 pp.

Hässel de Menéndez, G., Rubies, M. 2009. Catalogue of Marchantiophyta and Anthocerotophyta of southern South America. J. Cramer in der Gebr. Borntraeger Verlagsbuchhandlung, Berlin. 672 pp.

León, C.A., Oliván, G., Larraín. J., Vargas, R., Fuertes, E. 2014. Bryophytes and lichens in peatlands and Tepualia stipularis forest of Isla Grande de Chiloé-Chile. Anales del Jardín Botánico de Madrid 71(1): e003.

León-Vargas, Y., Engwald, S., Proctor, M.C.F. 2006. Microclimate, light adaptation and desiccation tolerance of epiphytic bryophytes in two Venezuelan cloud forests. Journal of Biogeography 33(5): 901-913.

Müller, F. 2009. An updated checklist of the mosses of Chile. Archives for Bryology 58: 1-124.

Pisano, E. 1977. Fitogeografía de Fuego-Patagonia Chilena. I. Comunidades vegetales entre las latitudes 52 y $56^{\circ} \mathrm{S}$. Anales del Instituto de la Patagonia 8: 121-250.

Promis, A., Bergh, G., Serra, M.T., Cruz, G. 2013. Descripción de la flora vascular en el sotobosque de un bosque pantanoso y de una pradera antropogénica húmeda de Juncus procerus en el valle del río Cisnes, Región de Aysén, Chile. Gayana Botánica 70(1): 164-169.

Ramírez, C., San Martín, C., San Martín, J. 1996. Estructura florística de los bosques pantanosos de Chile SurCentral. En: Armesto, J.J., Villagrán, C., Arroyo, M.T.K.
(Eds.), Ecología de los Bosques Nativos de Chile, pp. 215234. Editorial Universitaria, Santiago, Chile.

Rozzi, R., Armesto, J.J., Goffinet, B., Buck, W., Massardo, F., Silander, J., Arroyo, M.T.K., Russell, S., Anderson, C.B., Cavieres, L.A., Callicott, J.B. 2008. Changing lenses to assess biodiversity: Patterns of species richness in subAntarctic plants and implications for global conservation. Front. Ecol. Environ. 6(3): 131-137.

Schaumann, F. 2005. Terricolous bryophyte vegetation of Chilean temperate rain forests. Communities, adaptative strategies and divergence patterns. Studies in austral temperate rain forest bryophytes 26. Bryophytorum Bibliotheca 62: 1-153.

Shaw, A.J., Cox, C.J., Goffinet, B. 2005. Global patterns of moss diversity: taxonomic and molecular inferences. Taxón 54: 337-352.

Söderström, L., Hagborg, A., von Konrat, M., BartholomewBegan, S., Bell, D., Briscoe, L., Brown, E., Cargill, D.C., da Costa, D.P., Crandall-Stotler, B.J., Cooper, E.D., Dauphin, G., Engel, J., Feldberg, K., Glenny, D., Gradstein, S.R., He, X., Heinrichs, J., Hentschel, J., Ilkiu-Borges, A.L., Katagiri, T., Konstantinova, N.A., Larraín, J., Long, D., Nebel, M., Pócs, T., Puche, F., Reiner-Drehwald, E., Renner, M., SassGyarmati, A., Schäfer-Verwimp, A., Segarra-Moragues, J.G., Stotler, R.E., Sukkharak, P., Thiers, B., Uribe, J., Váňa, J., Villarreal, J., Wigginton, M., Zhang, L., Zhu, R.L. 2016. World checklist of hornworts and liverworts. PhytoKeys 59: 1-828.

Stapanian, M.A., Schumacher, W., Gara, B., Viau, N. 2016. Mosses in Ohio wetlands respond to indices of disturbance and vascular plant integrity. Ecological Indicators 63: 110-120.

Stewart, K.J., Mallik, A.U. 2006. Bryophyte responses to microclimatic edge effects across riparian buffers. Ecological Applications 16(4): 1474-1486.

Tropicos.org (2019). Missouri Botanical Garden. URL: http:// legacy.tropicos.org/ Accedido: Octubre 19, 2018.

Villagrán, C. 2018. Biogeografía de los bosques subtropicaltemplados del sur de Sudamérica. Hipótesis históricas. Magallania 46(1): 27-48.

Villagrán, C., Barrera, E. 2002. Musgos del Archipiélago de Chiloé, Chile. Corporación Nacional Forestal, Gobierno de Chile. Puerto Montt, Chile. 24 pp.

Villagrán, C., Barrera, E., Medina, C. 2002. Las hepáticas del Archipiélago de Chiloé, Chile. Corporación Nacional Forestal, Gobierno de Chile. Puerto Montt, Chile. 26 pp.

Villagrán, C., Hässel de Menéndez, G., Barrera, E. 2005. Hepáticas y Anthocerotes del Archipiélago de Chiloé. Una introducción a la flora briofítica de los ecosistemas templados lluviosos del sur de Chile. Corporación de Amigos del Museo Nacional de Historia Natural, Santiago, Chile. 160 pp.

Received: 18.06 .2020

Accepted: 27.05.2020 
Anexo 1. Catálogo de las briófitas del bosque pantanoso (BP) y la pradera antropogénica húmeda de Juncus procerus (PHJ). / List of the bryophyte species in the swamp forest (BP) and the anthropogenic wet prairie of Juncus procerus (PHJ).

\begin{tabular}{|c|c|c|c|c|c|c|c|}
\hline DIVISIÓN/FAMILIA & Nombre Científico & M & FV & PD-G & PD-L & $\mathbf{S}$ & FMV \\
\hline \multicolumn{8}{|c|}{ ANTHOCEROTOPHYTA } \\
\hline Dendrocerotaceae & Dendroceros cichoraceus (Mont.) Gottsche & $T$ & ET & $\mathrm{E}$ & VNS & $\mathrm{R}$ & $\mathrm{BP}$ \\
\hline \multicolumn{8}{|l|}{ BRYOPHYTA } \\
\hline Polytrichaceae & Dendroligotrichum dendroides (Brid. ex Hedw.) Broth & $\mathrm{PL}$ & $\mathrm{DE}$ & $\mathrm{E}$ & VNS-JF & $\mathrm{S}$ & BP-PHJ \\
\hline \multirow[t]{4}{*}{ Dicranaceae } & Dicranoloma billarderii (Brid.) Paris & A & $\mathrm{CL}$ & PAN & MVNS-JF & $\mathrm{R}$ & PHJ \\
\hline & Dicranoloma chilense (De Not.) Ochyra \& Matteri & A & $\mathrm{CC}$ & E & VNS-JF & $\mathrm{R}$ & PHJ \\
\hline & Dicranoloma imponens (Mont.) Renauld & $A$ & $\mathrm{CL}$ & A & VNS & C & BP-PHJ \\
\hline & Dicranoloma robustum (Hook. f. \& Wilson) Paris & $A$ & $\mathrm{CC}$ & PAN & VNS & $\mathrm{H}$ & $\mathrm{PHJ}$ \\
\hline Bartramiaceae & Breutelia dumosa Mitt. & $A$ & $\mathrm{CL}$ & $\mathrm{E}$ & VNS & $\mathrm{S}$ & $\mathrm{PHJ}$ \\
\hline \multirow[t]{3}{*}{ Orthotrichaceae } & Ulota macrocalycina Mitt. & $A$ & $\mathrm{CC}$ & $\mathrm{E}$ & VNS & $\mathrm{R}$ & $\mathrm{PHJ}$ \\
\hline & Zygodon pentastichus (Mont.) Müll. Hal. & A & $\mathrm{CC}$ & $\mathrm{E}$ & MVNS-JF & C & PHJ \\
\hline & Zygodon pichinchensis (Taylor) Mitt. & A & $\mathrm{CC}$ & $\mathrm{E}$ & VNS & C & PHJ \\
\hline Rhizogoniaceae & $\begin{array}{l}\text { Hymenodontopsis mnioides (Hook.) N.E. Bell, } \\
\text { A.E. Newton \& D. Quandt }\end{array}$ & A & $\mathrm{CL}$ & B & MVNS-JF & $\mathrm{s}$ & BP-PHJ \\
\hline Hypnodendraceae & Hypnodendron microstictum Mitt. ex A. Jaeger \& Sauerb. & PL & DE & $\mathrm{E}$ & MVNS-JF & C & $\mathrm{BP}$ \\
\hline \multirow[t]{2}{*}{ Ptychomniaceae } & Ptychomniella ptychocarpa (Schwägr.) W.R. Buck, C. J. Cox, A.J. Shaw \& Goffinet & PL & $\mathrm{C}$ & $\mathrm{E}$ & MVNS-JF & C & $\mathrm{BP}$ \\
\hline & Ptychomnion cygnisetum (Müll. Hal.) Kindb. & PL & $\mathrm{CC}$ & $\mathrm{E}$ & XMVNS-JF & $\mathrm{S}$ & $\mathrm{BP}-\mathrm{PHJ}$ \\
\hline Hypopterygiaceae & Arbusculohypopterigium arbuscula (Brid.) M. Stech, T. Pfeiff. \& W. Frey & PL & DE & $\mathrm{E}$ & MVNS-JF & C & $\mathrm{BP}-\mathrm{PHJ}$ \\
\hline Daltoniaceae & Distichophyllum dicksonii (Hook. \& Grev.) Mitt. & PL & $\mathrm{CC}$ & $\mathrm{E}$ & VNS & C & $\mathrm{BP}$ \\
\hline Amblystegiaceae & Sanionia uncinata (Hedw.) Loeske & PL & $\mathrm{CL}$ & B & VNS & $\mathrm{S}$ & $\mathrm{BP}-\mathrm{PHJ}$ \\
\hline Rigodiaceae & Rigodium pseudothuidium Dusén & PL & $\mathrm{T}$ & $\mathrm{E}$ & XMVNS-JF & C & $\mathrm{BP}$ \\
\hline Neckeraceae & Porothamnium arbusculans (Müll. Hal.) M. Fleisch. & PL & DE & $\mathrm{E}$ & MVNS-JF & $\mathrm{S}$ & $\mathrm{BP}$ \\
\hline Lembophyllaceae & Acrocladium auriculatum (Mont.) Mitt. & PL & $\mathrm{T}$ & C & VNS & C & $\mathrm{PHJ}$ \\
\hline \multicolumn{8}{|c|}{ MARCHANTIOPHYTA } \\
\hline Adelanthaceae & Syzygiella colorata (Lehm.) K. Feldberg, Váňa, Hentschel et Heinrichs & $\mathrm{F}$ & $\mathrm{CC}$ & ANZAF & VNS-ChC-JF & C & $\mathrm{BP}-\mathrm{PHJ}$ \\
\hline Cephaloziellaceae & Cephaloziella hispidissima R.M.Schust. & $\mathrm{F}$ & $\mathrm{EF}$ & NZ & VNS & C & $\mathrm{PHJ}$ \\
\hline Lophoziaceae & Andrewsianthus australis J.J. Engel & $\mathrm{F}$ & $\mathrm{T}$ & $\mathrm{E}$ & VNS & C & $\mathrm{PHJ}$ \\
\hline
\end{tabular}




\begin{tabular}{|c|c|c|c|c|c|c|c|}
\hline DIVISIÓN/FAMILIA & Nombre Científico & M & FV & PD-G & PD-L & S & FMV \\
\hline Balantiopsidaceae & Isotachis humectata (Hook.f. et Taylor) Steph. & $\mathrm{F}$ & $\mathrm{CL}$ & $\mathrm{E}$ & VNS-ChC & $S$ & $\mathrm{PHJ}$ \\
\hline Blepharidophyllaceae & Blepharidophyllum densifolium (Hook.) Ångstr. ex C. Massal. & $\mathrm{F}$ & $\mathrm{CL}$ & $\mathrm{E}$ & VNS & $\mathrm{S}$ & $\mathrm{PHJ}$ \\
\hline Lepicoleaceae & Lepicolea ochroleuca (Spreng.) Spruce & $\mathrm{F}$ & A & $\mathrm{AF}$ & VNS-ChC-JF & $\mathrm{C}$ & BP- $\mathrm{PHJ}$ \\
\hline \multirow[t]{7}{*}{ Lepidoziaceae } & Acromastigum cunninghamii (Steph.) A.Evans & $\mathrm{F}$ & $\mathrm{T}$ & NZ & VNS & $C$ & $\mathrm{PHJ}$ \\
\hline & Bazzania peruviana (Nees) Trevis. & $\mathrm{F}$ & $\mathrm{T}$ & $\mathrm{N}$ & VNS-JF & $\mathrm{S}$ & BP \\
\hline & Bazzania nitida (F. Weber) Grolle & $\mathrm{F}$ & $\mathrm{T}$ & PAN & VNS & $\mathrm{C}$ & BP \\
\hline & Lepidozia chordulifera Taylor & $\mathrm{F}$ & A & $\mathrm{E}$ & VNS-ChC-JF & $\mathrm{C}$ & BP- $\mathrm{PHJ}$ \\
\hline & Neolepidozia seriatitexta (Steph.) J.J. Engel & $\mathrm{F}$ & $\mathrm{T}$ & $\mathrm{E}$ & VNS-JF & $\mathrm{C}$ & BP- $\mathrm{PHJ}$ \\
\hline & Telaranea blepharostoma (Steph.) Fulford & $\mathrm{F}$ & $\mathrm{EF}$ & $\mathrm{E}$ & VNS-ChC-JF & $C$ & BP- $\mathrm{PHJ}$ \\
\hline & Hyalolepidozia bicuspidata (C. Massal.) S. W. Arnell ex Grolle & $\mathrm{F}$ & $\mathrm{EF}$ & $\mathrm{AF}$ & VNS-R-JF & $\mathrm{R}$ & BP \\
\hline \multirow[t]{9}{*}{ Lophocoleaceae } & Clasmatocolea ctenophylla (Schiffn.) Grolle & $\mathrm{F}$ & ES & $\mathrm{E}$ & VNS & $\mathrm{C}$ & $\mathrm{PHJ}$ \\
\hline & Clasmatocolea fulvella (Hook.f. et Taylor) Grolle & $\mathrm{F}$ & $\mathrm{CC}$ & $\mathrm{E}$ & VNS & $\mathrm{S}$ & BP \\
\hline & Clasmatocolea gayana (Mont.) Grolle & $\mathrm{F}$ & ES & $\mathrm{E}$ & VNS & $C$ & BP- $\mathrm{PHJ}$ \\
\hline & Clasmatocolea moniliformis J.J.Engel & $\mathrm{F}$ & ES & $\mathrm{E}$ & VNS & $C$ & $\mathrm{PHJ}$ \\
\hline & Heteroscyphus montagnei (Steph.) Fulford & $\mathrm{F}$ & ES & $\mathrm{E}$ & VNS-JF & $\mathrm{S}$ & BP- $\mathrm{PHJ}$ \\
\hline & Heteroscyphus valdiviensis (Mont.) Schiffn & $\mathrm{F}$ & ES & $\mathrm{E}$ & VNS & C & BP \\
\hline & Lophocolea hahnii Steph. & $\mathrm{F}$ & ES & $\mathrm{E}$ & VNS & C & BP \\
\hline & Lophocolea muricata (Lehm.) Nees & $\mathrm{F}$ & ES & $\cos$ & VNS-ChC-JF & $\mathrm{S}$ & BP \\
\hline & Lophocolea sylvatica Mitt & $\mathrm{F}$ & ES & $\mathrm{E}$ & VNS & $\mathrm{S}$ & BP \\
\hline \multirow[t]{6}{*}{ Plagiochilaceae } & Plagiochila chonotica Taylor & $\mathrm{F}$ & A & $\mathrm{E}$ & VNS & C & BP- PHJ \\
\hline & Plagiochila dura De Not. & $\mathrm{F}$ & A & $\mathrm{E}$ & VNS & C & BP \\
\hline & Plagiochila hookeriana Lindenb. & $\mathrm{F}$ & A & E & VNS-JF & $\mathrm{S}$ & BP- PHJ \\
\hline & Plagiochila lophocoleoides Mont. & $\mathrm{F}$ & A & $\mathrm{E}$ & VNS & $\mathrm{H}$ & BP \\
\hline & Plagiochila rubescens (Lehm. et Lindenb.) Lindenb. & $\mathrm{F}$ & A & $\mathrm{E}$ & VNS-ChC-JF & C & BP- PHJ \\
\hline & Plagiochila subpectinata Besch. et C. Massal. & $\mathrm{F}$ & A & $\mathrm{E}$ & VNS-R & C & BP \\
\hline Trichocoleaceae & Leiomitra elegans (Lehm.) Hässel & $\mathrm{F}$ & $\mathrm{T}$ & ANZAF & VNS-JF & $\mathrm{H}$ & BP- $\mathrm{PHJ}$ \\
\hline Schistochilaceae & Schistochila stratosa (Mont.) A. Evans & $\mathrm{F}$ & A & $\mathrm{E}$ & VNS-JF & $\mathrm{H}$ & BP \\
\hline \multirow[t]{2}{*}{ Frullaniaceae } & Frullania chilensis Steph. & $\mathrm{F}$ & ES & $\mathrm{E}$ & VNS-ChC-JF & C & PHJ \\
\hline & Frullania magellanica F. Weber \& Nees & $\mathrm{F}$ & ES & $\mathrm{E}$ & VNS & C & $\mathrm{PHJ}$ \\
\hline Lejeuneaceae & Cheilolejeunea obtruncata (Mont.) Solari & $\mathrm{F}$ & ES & E & VNS & C & BP- PHJ \\
\hline
\end{tabular}




\begin{tabular}{|c|c|c|c|c|c|c|c|}
\hline DivisIÓN/FAMILIA & Nombre Científico & M & FV & PD-G & PD-L & S & FMV \\
\hline & Lejeunea hahnii Solari & $\mathrm{F}$ & ES & $\mathrm{E}$ & VNS & $\mathrm{R}$ & $\mathrm{BP}$ \\
\hline Lepidolaenaceae & Gackstroemia magellanica (Lam.) Trevis. & $\mathrm{F}$ & A & $\mathrm{E}$ & VNS-ChC-JF & C & $\mathrm{PHJ}$ \\
\hline Porellaceae & Porella subsquarrosa (Nees et Mont.) Trevis. & $\mathrm{F}$ & ES & $\mathrm{E}$ & VNS-ChC-JF & $\mathrm{C}$ & $\mathrm{PHJ}$ \\
\hline \multirow[t]{2}{*}{ Aneuraceae } & Riccardia prehensilis (Hook.f. et Taylor) C. Massal. & TS & A & $\mathrm{E}$ & VNS & $\mathrm{S}$ & $\mathrm{BP}$ \\
\hline & Riccardia spinulifera C. Massal. & TS & ET & $E$ & VNS & $\mathrm{C}$ & $\mathrm{BP}$ \\
\hline \multirow[t]{2}{*}{ Metzgeriaceae } & Metzgeria frontipilis (Lindb.) Kuwah. \& J.J. Engel & $\mathrm{F}$ & $\mathrm{DE}$ & $E$ & VNS & $\mathrm{S}$ & $\mathrm{PHJ}$ \\
\hline & Metzgeria sp. & TS & ET & $\mathrm{D}$ & $\mathrm{D}$ & $\mathrm{H}$ & $\mathrm{BP}$ \\
\hline Pallaviciniaceae & Pallavicinia xiphoides (Hook. f. et Taylor) Trevis. & TS & $\mathrm{DE}$ & ANZ & VNS-JF & $\mathrm{S}$ & BP- $\mathrm{PHJ}$ \\
\hline
\end{tabular}

$M=$ morfología $(A=$ Acrocarpo; $F=$ Foliosa; $P L=$ Pleurocarpo; $T$ = Talosa; $T S=$ Talosa simple). $F V=$ forma de vida $(A=A b a n i c o ; C=C o l g a n t e s ; C C=$ césped corto $\mathrm{CL}=$ césped largo; $\mathrm{DE}=$ Dendroide; $\mathrm{EF}=$ Estera filiforme; $\mathrm{ES}=$ Estera suave; $\mathrm{ET}=$ Estera taloide; $\mathrm{T}=$ Tramas). $\mathrm{PD}-\mathrm{G}=$ patrones de distribución global $(\mathrm{A}=\mathrm{Austral}$ antártico; $\mathrm{AF}=$ Compartida con Sudáfrica; $\mathrm{ANZ}=$ Compartida con Australia y Nueva Zelanda; ANZAF = Compartida con Australia, Nueva Zelanda y Sudáfrica; B = Bipolar; C = Circumsubantártico; COS = Cosmopolita; D = Desconocido; $E$ = Endémico de Sudamérica; $N$ = Neotropical; NZ = Compartida con Nueva Zelanda; PAN = Pantropical). $\mathrm{PD}-\mathrm{L}$ = patrones de distribución local (A = Austral; $\mathrm{ChC}=$ Disyunto con Chile Central; $\mathrm{D}=\mathrm{Desconocido}$; JF = Disyunto con Juan Fernández; $\mathrm{M}=$ Mediterráneo; $\mathrm{N}=$ Nordpatagónico; $\mathrm{R}=$ Disyunto con Fray Jorge; $\mathrm{S}=$ Subantártico; $\mathrm{V}=$ Valdiviano; $\mathrm{X}=$ Xeromórfico). $\mathrm{S}=$ sustrato ( $\mathrm{C}=$ corteza de árboles; $\mathrm{H}=$ hojas; $\mathrm{R}=$ ramas; $\mathrm{S}$ = suelo). $\mathrm{FMV}=$ formación vegetal ( $\mathrm{BP}=$ bosque pantanoso; $\mathrm{PHJ}=$ pradera antropogénica húmeda de Juncus procerus). 